\title{
A LIBRARY OF EMBEDDED PLATFORM COMPONENTS FOR THE SIMULATION OF REAL-TIME EMBEDDED SYSTEMS
}

\author{
Joost Mertens \\ Ken Vanherpen \\ Joachim Denil \\ Paul De Meulenaere \\ CoSys-Lab (FTI) \\ University of Antwerp \\ Antwerp, Belgium \\ joost.mertens@ student.uantwerpen.be \\ $\{$ ken.vanherpen, joachim.denil, paul.demeulenaere\}@uantwerpen.be
}

\begin{abstract}
When modeling the control algorithm(s) of a Cyber-Physical System, control engineers evaluate if its behavior satisfies the specifications by executing a Model-in-the-Loop simulation. However, often the deployment of a control algorithm on an embedded system affects its behavior. Tools exist that include these deployment effects in the Model-in-the-Loop simulation. However, they require engineers to significantly modify existing control models to incorporate these effects at this (higher) abstraction level. As such, engineers are restricted in evaluating various deployment configurations. We created a library of configurable embedded components that enables an engineer to model the embedded platform at the same level of abstraction as the model of the control algorithm. Our method requires minimal changes to the modeled control algorithm so that evaluating its deployed behavior, using a co-simulation, is facilitated. We compared our co-simulation results with a deployed system and evaluated our approach with current state-of-the-art tools.
\end{abstract}

Keywords: Control Embedded Co-Design, Cyber-Physical Systems, Model-Based Systems Engineering, Real-Time Simulation, SimEvents ${ }^{\circledR}$

\section{INTRODUCTION}

Cyber-Physical Systems (CPS) are systems in which control algorithms running on an (distributed) embedded platform are tightly coupled with physical systems through a feedback loop. Physical refers to the physical processes to be controlled, also called the plant, whilst cyber refers to the computational power and control introduced by an embedded system in combination with sensors and actuators (Lee 2008). Applications range from driving aids in cars, such as anti-lock brakes and adaptive cruise control, to medical devices and robotics (Lee 2008).

The design of a CPS is often typed by a multi-disciplinary design team that follows a well-defined modelbased development process. The process starts by a common activity in which the functional requirements (i.e., specifications) and architecture of the system are defined. The mechanical engineer initiates the design process by reasoning over the physics of the system and, subsequently, constructing a model of the plant. Given the plant model, the control engineer models a control algorithm using automata-based and/or flowbased formalisms. To verify the behavior of the modeled control algorithm, a Model-in-the-Loop (MiL) 
simulation is executed in which the plant model is linked to the model of the control algorithm using a feedback loop. If the result of the MiL simulation satisfies the specifications, the modeled control algorithm is prepared for deployment on a computational platform, often an embedded system. Therefore, the control algorithm is divided into a set of software components (SWC) that are typically executed by a set of tasks on a Real-Time Operating System (RTOS).

When verifying the specifications of the deployed system, differences can be observed between the deployed and simulated behavior of the control algorithm. One contributing factor is the fact that simulated models are executed by the Zero Execution Time (ZET) principle (Derler et al. 2013). As shown on the left-hand side of Figure 1, models executed using this principle sample their inputs $(a)$, compute (part of) the model $(b)$, and actuate the outputs $(c)$ in one and the same time step. When comparing this principle with the actual execution of a SWC, shown in the middle part of Figure 1, we observe that these three steps do take a finite amount of time. Assuming periods $a$ and $c$ are sufficiently short, the simulation of a modeled control algorithm is better approximated by the Logical Execution Time (LET) principle (Derler et al. 2013) in which a certain amount of time is considered between input sampling and the actuation of the outputs.

Other contributing factors explaining the differences between simulated and deployed are the fact that in simulation all sections of the model are simulated concurrently, feature floating-point precision and experience no communication delays. By contrast, the deployed algorithm $(i)$ is executed on a processor by an RTOS in which tasks have priorities, (ii) possibly uses fixed-point precision, and (iii) is possibly distributed over a set of microcontrollers. It has been shown in (Cervin et al. 2003) how these non-ideal factors contribute to degraded controller performance. It is therefore desirable to take the effects of the embedded platform into account when verifying the behavior of a modeled control algorithm. Tools that enable this already exist and might be provided as toolboxes for commonly used modeling environments like Simulink $^{\circledR}$ (MathWorks 2019c). Well known examples are TrueTime (Cervin et al. 2003, Henriksson et al. 2003) and, more recently, T-Res (Cremona et al. 2015). Unfortunately, these tools either don't integrate well with existing Simulink ${ }^{\circledR}$ models, requiring one to generate code from the control model, or they are limited in their applicability by not supporting distributed algorithms.

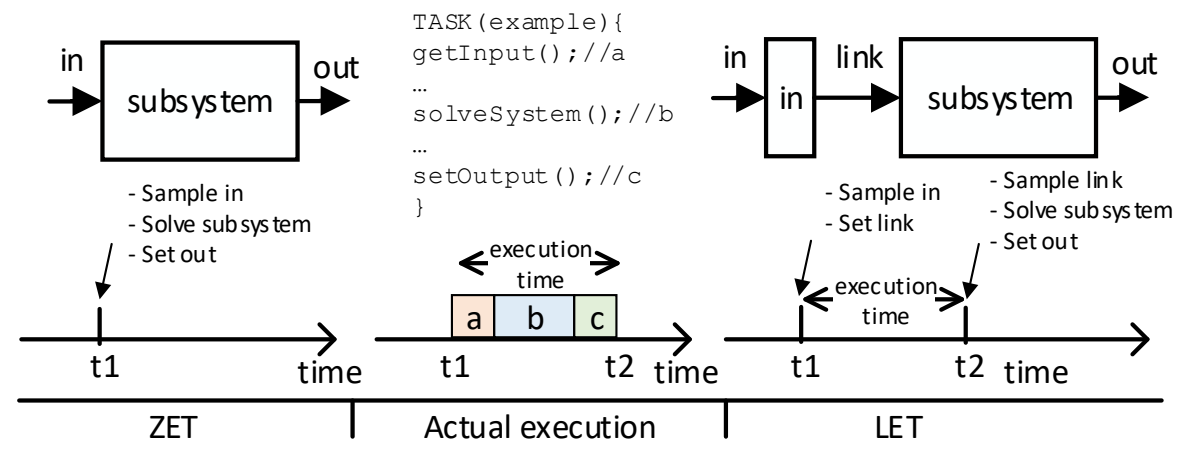

Figure 1: ZET and LET (Derler et al. 2013) compared to the deployed execution.

In this paper, we demonstrate a Simulink ${ }^{\circledR}$ library of configurable components that represents those parts of the embedded platform that might influence the behavior of the control model. It enables an embedded engineer to model the embedded platform at an abstraction level that facilitates integration with the control model. Similar to T-Res, our approach eliminates the need to generate code from the control model, achieving tight integration with Simulink ${ }^{\circledR}$. The library removes the reliance on external scheduling and network simulators by relying on SimEvents ${ }^{\circledR}$ (MathWorks 2019b), a discrete event simulator in Simulink ${ }^{\circledR}$. We val- 
idated the library by comparing the behavior of a control model annotated with library blocks to the behavior of a realized system using an electric power window controller as a case study.

The remainder of this paper is structured as follows: In section 2, related work regarding the simulation of deployment effects is discussed. Then, in section 3, we detail the development of the library while its use is discussed in section 3.3. In section 4, we discuss how the presented library compares to the use of TrueTime when evaluating the deployed control behavior at a higher abstraction level. Finally, section 5 concludes the paper and presents future research directions.

\section{RELATED WORK}

As already mentioned, different tools exist to assist engineers in evaluating the deployed behavior of a control algorithm at a higher level of abstraction, i.e., at the control model level. TrueTime by Cervin et al. (2003) is a Simulink ${ }^{\circledR}$ toolbox that includes a kernel block and networking blocks. The kernel block is used to execute a control algorithm as if it were scheduled by an RTOS by supporting task preemption, interrupts and multiple scheduling policies. Each task is further divided in segments representing sections of a task that execute sequentially. The segments allow for fine grained control of each task. It is required to include the control algorithm in either Matlab ${ }^{\circledR}$ scripts, or C++ code. In other words, code needs to be generated from the modeled control algorithm. The networking blocks can be used to simulate delays and loss probabilities introduced by various types of networks.

T-Res by Cremona et al. (2015) improves on the integration with existing Simulink ${ }^{\circledR}$ models, by using triggered subsystems instead of generated code. Similar to the TrueTime approach, a kernel block determines the execution order and execution times of different tasks which are in turn split in segments. Each segment is coupled to a subsystem modeled in Simulink ${ }^{\circledR}$. Since segments are executed sequentially, the linked subsystems are also executed sequentially. The kernel, task and network blocks are explicitly modeled as Simulink ${ }^{\circledR}$ blocks that can be configured through a set of block parameters. For the scheduling and network simulations, T-Res relies on external simulators that interface with Simulink through the aforementioned blocks.

Vanherpen et al. (2015) describe a round trip method which allows a Simulink ${ }^{\circledR}$ control model to be back annotated with additional delay blocks that reflect the Worst-Case Execution Time (WCET) and Scheduling Time (ST) of the deployed control algorithm. Their method can be summarized in 3 steps: design a control model, execute a schedulability analysis (or measure the deployed model) and annotate the control model with the timing related deployment effects. These annotations transform the model from a ZET representation to a LET representation. The result is that the control engineer can evaluate the deployed behavior and tune the control algorithm at their level of abstraction without overloading the control algorithm with additional blocks.

Denil et al. (2017) show how the Discrete EVent System specification (DEVS) formalism (Zeigler 1976) can be used to model and evaluate AUTOSAR (AUTOSAR 2018) based systems. In their work, DEVS is used to model the AUTOSAR software and a CAN bus. A power window and ABS case study show how task execution of the AUTOSAR operating system can be observed. Calibration of the model is done by annotating the code, deploying it and measuring the execution times. The simulation enables the engineer to observe the effects of deployment and networking on the control algorithm.

Li et al. (2016) elaborate on how the SimEvents ${ }^{\circledR}$ discrete event library in Simulink ${ }^{\circledR}$ can be used to simulate a multi-core scheduler. They note that by using SimEvents ${ }^{\circledR}$, a true discrete-event simulator is used instead of relying on variable step simulation with zero-crossings. They further note that in the SimEvents ${ }^{\circledR}$ approach, the algorithmic and computational part of the simulation are separated from each other. In that respect, Brandberg and Di Natale (2018) demonstrate how SimEvents ${ }^{\circledR}$ models can be used to simulate scheduling 
and memory access delays in multi-core systems. Using SimEvents ${ }^{\circledR}$ they simulate a benchmark provided by Bosch and demonstrate that it is possible to perform an AUTOSAR compatible LET simulation. They also draw comparisons to TrueTime and T-Res and note how using SimEvents ${ }^{\circledR}$ allows for better simulation performance.

\section{A LIBRARY OF CUSTOMIZABLE COMPONENTS REPRESENTING THE EMBEDDED PLATFORM}

In order to facilitate the simulation of control models using the LET principle, we aim to design a set of components that model those aspects of the embedded platform that highly affect the performance of the control algorithm. While doing so, we assumed the following:

- Model-Based Systems Engineering (MBSE) is used in which both the mechanical components and the control algorithms are modeled. For the latter we assume the use of the Simulink ${ }^{\circledR}$ modeling environment.

- A component-based approach is used. The control algorithm is subdivided into a set of (reusable) components. This subdivision is decided during a common architectural design step allowing for a mapping of control components onto RTOS tasks.

- The execution time of the different RTOS tasks is known in advance, either through measurements or through timing analysis.

Transforming a ZET based simulation model to a LET based model can be achieved in different ways. One approach is to introduce delay blocks containing execution time and scheduling time, as done in (Vanherpen et al. 2015). However, this method does not introduce a scheduled execution of the controller. Another approach is to introduce tasks and segments as done by TrueTime and T-Res. The tasks are executed by a scheduler with a configurable scheduling policy. Each segment represents a distinct part of the task. In LET a task must have at least three segments: input sampling, calculations, output setting. In TrueTime the segmentation is script based, whilst in T-Res each segment triggers the execution of a triggered subsystem. The segmentation approach of T-Res has a minimal impact on the control engineer's view on the system and fits the model-based assumption the best. Therefore, we decide to use the same approach, but instead of using triggered subsystems, we use Simulink ${ }^{\circledR}$ functions, as demonstrated in (Li et al. 2016).

These assumptions and design choice on how to implement a LET based model lead to a library of configurable hardware and software components that represent those parts of the embedded platform that influence the performance of the deployed control model. This includes RTOS related software components (e.g., task execution, scheduling policy, interrupts) and hardware components (e.g., networking delays and quantization effects). We use the most appropriate formalism for these blocks: either discrete events or causal block diagrams (CBD). For the later, we rely on Simulink ${ }^{\circledR}$ while SimEvents ${ }^{\circledR}$ is used to model the discrete events aspects. The next paragraph aims to briefly introduce SimEvents ${ }^{\circledR}$.

SimEvents ${ }^{\circledR}$ is a discrete event simulator in which entities flow until termination. Figure 2 illustrates a model in which the most common SimEvents ${ }^{\circledR}$ blocks are used. Entities are generated by Entity Generator blocks and terminated by Entity Terminator blocks. Entities have a set of attributes, which type the entity. Throughout their lifetime, entities can acquire resources through Resource Acquire blocks, be served by Entity Server blocks and be queued by Entity Queue blocks. Entity Input/Output Switch blocks are used to route the flow of entities. Being a discrete event simulator, only the discrete events in the system are simulated, i.e., generation of entities, arrival/departure of entities to/from a block, completion of service in an Entity Server, entities being blocked and termination of entities. These events are collected on the Event Calendar. The Event Calendar determines the execution order of events and is sorted chronologically. 


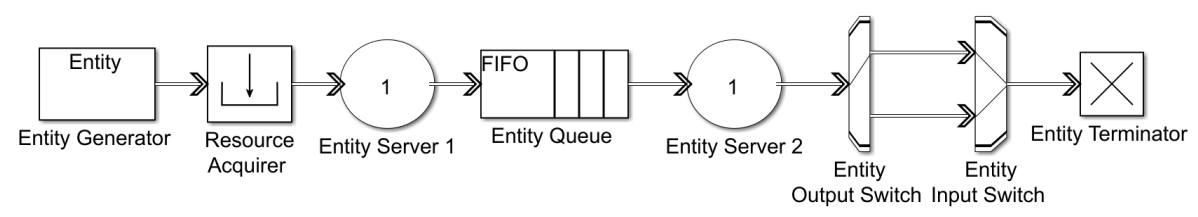

Figure 2: SimEvents chart with the most commonly used blocks.

Entities are completely generic. As such, they can be used to represent people shopping at a grocery store or controller tasks being scheduled by an operating system.

\subsection{RTOS Related Software Components}

Our library intends to model the embedded platform at a higher abstraction level (i.e., the one of the control engineer) while maintaining lower level properties that potentially influence the behavior of the control algorithm. When evaluating current implementations, both TrueTime and T-Res rely on a generic task scheduler, complicating the configuration for the embedded engineer as certain concepts of the RTOS may not exist in TrueTime or T-Res. In contrast to previous work, we create a library based on an existing RTOS specification instead of a generic specification. In particular, the blocks related to the RTOS are based on the OSEK/VDX specification (OSEK/VDX 2005). This enables the embedded engineer to obtain a one-to-one relation between the parameters of the embedded platform model and the actual deployment configuration settings. Given our analysis of software related aspects that might influence the control behavior, the RTOS library implements the following aspects of the OSEK/VDX specification:

- Task Management: Tasks, Scheduling and Processing.

- Events: used for synchronization purposes.

- Resources: essentially mutexes, in which a resource can be a peripheral device or shared memory.

- Interrupt Service Routines (ISR)

- Alarms

As these elements can be considered to be parts of a Discrete Event System (DES), SimEvents ${ }^{\circledR}$ is used to model the RTOS related software components. Figure 3 shows the main blocks of the RTOS library. Event and resource management aspects of the RTOS do not have an explicit representation as they are implemented by the Task Processor block. The blocks are in essence wrappers around a model that consists of generic SimEvents ${ }^{\circledR}$ entities. The wrapper enables the configuration of the blocks using a pre-defined set of configurable parameters. As an example, we discuss the implementation of the Task block in more detail.

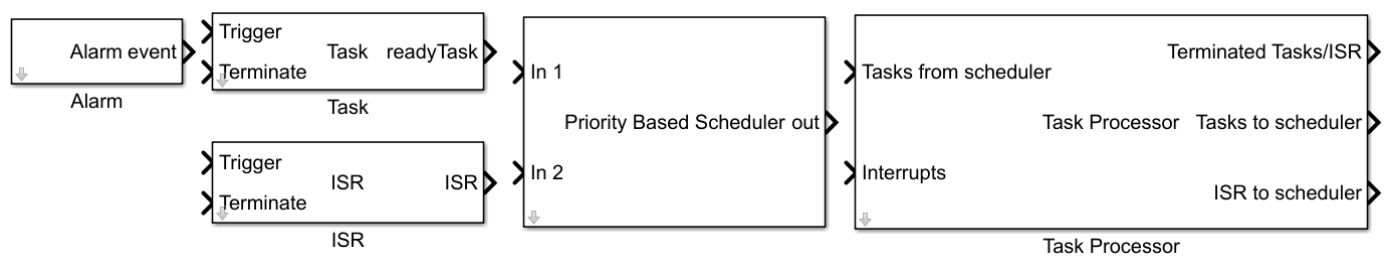

Figure 3: Main RTOS library blocks. 
The internal view of the Task block is shown in figure 4, its parameter dialog in figure 5. We start at the left-hand side, where an Entity Generator periodically generates a new task entity. Alternatively, instead of periodic generation, an entity can also be generated by an event. In that case the block gains an additional Trigger input as can be seen in figure 3. Every task has a set of parameters, such as Task ID and Task Priority that can be configured through the parameter dialog. The parameter dialog also allows one to configure the execution time of the task segments, the resource usage and event synchronization of each segment and whether or not the task is preemptable. The generated entities flow into an Entity Queue labeled Activation Queue in which multiple activations of the task can be queued. In case this queue is full, the tasks flow to the Entity Terminator instead. Each task allows multiple activations up to the Maximum Activation Count parameter of the block, however only one activation can be actively flowing through the system at a time. The Entity Gate ensures this is the case. This block is either open, allowing entities to flow through, or closed, blocking entities at its input. By default the gate is open. Once the first task passes through Entity Server 1, the gate is closed by calling Simulink function controlGate $(u)$. The task then flows out through readyTask port. Once a task has finished execution, i.e., all its segments have been handled by the Task Processor block, it is routed back through the input port Terminate. It enters Entity Server 2, which opens the Entity Gate, allowing a new task to pass through. The terminating task is then released from Entity Server 2 and terminated.

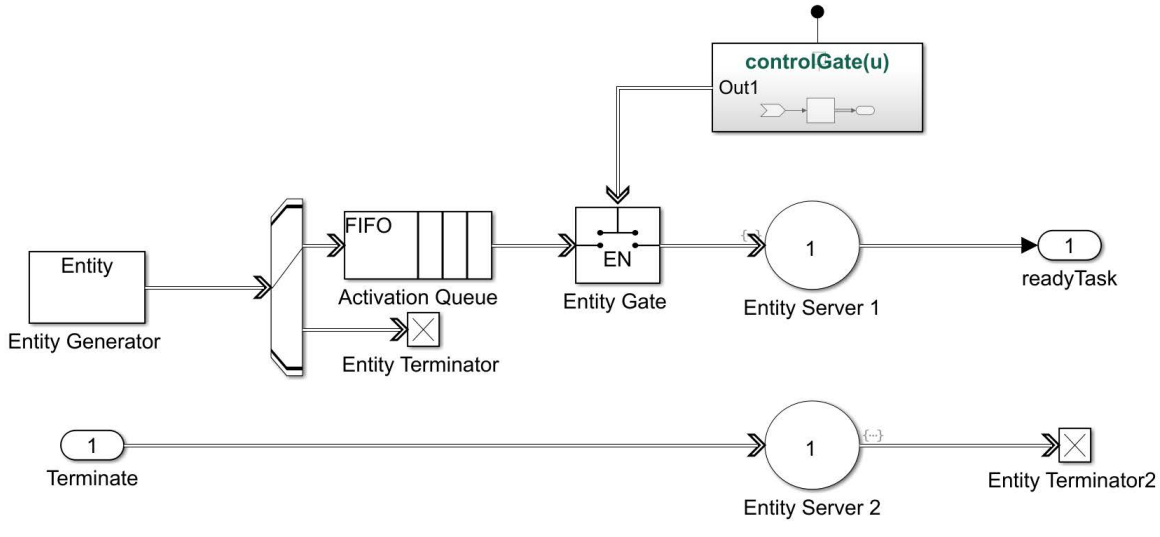

Figure 4: Internal view of the Task block.

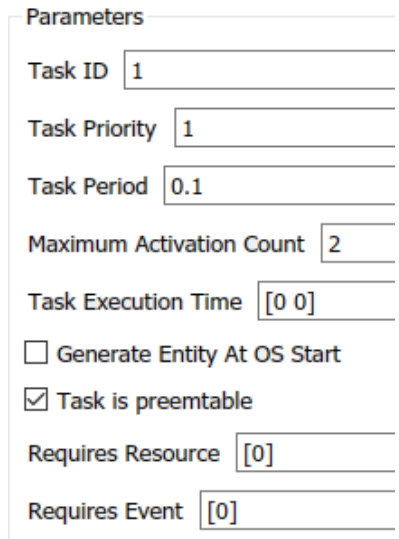

Figure 5: Task block parameter dialog.

\subsection{Hardware Components}

Besides RTOS related software components, some hardware effects might also influence the behavior of the control algorithm. These effects can be classified as delay effects or precision effects. Delay effects relate to the finite speed of networking peripherals. Precision relates to the finite precision that can be achieved by peripherals. Based on the available peripherals on the used microcontroller (Atmel Atmega328P), we modeled to following list of hardware components using either Causal Block Diagrams and/or SimEvents ${ }^{\circledR}$ :

- Analog to Digital Converter (ADC): The $A D C$ block simulates the conversion of analog inputs to digital outputs. The $A D C$ has a finite resolution and a finite conversion time, placing an upper bound on the number of conversions per second. For its model, we rely on Causal Block Diagrams. 
- Digital to Analog Converter (DAC): The DAC performs the opposite conversion, converting a digital input to an analog output. Just as the $A D C$ it has a finite conversion time. Again, we rely on Causal Block Diagrams for its implementation.

- Universal Synchronous/Asynchronous Receiver/Transmitter (USART): The USART allows for the simulation of serial point to point transmissions. Multiprocessor mode, in which multiple microcontrollers connect to a single serial link, is also supported. For its model, we rely on SimEvents ${ }^{\circledR}$.

- Serial Peripheral Interface (SPI): The SPI blocks can be used to simulate SPI master-slave communication, in which a single master device communicates with one or more slave devices. The implementation is obtained by relying on SimEvents ${ }^{\circledR}$.

- Controller Area Network (CAN): In the case of the CAN bus, we started from existing CAN Transceiver and CAN Bus blocks by (MathWorks 2019a). This SimEvents ${ }^{\circledR}$ based model was improved to also simulate CAN bit stuffing in order to achieve a more accurate model.

- FlexRay: The FlexRay blocks consist of a FlexRay Bus and FlexRay Transceiver block. Using these blocks, a single channel FlexRay bus can be simulated. Internally, the blocks consist of SimEvents ${ }^{\circledR}$ library blocks for the packet transmission, and a Stateflow ${ }^{\circledR}$ chart for keeping the FlexRay bus cycle. The FlexRay Bus block is used to configure the entire bus cycle. Again, we rely on SimEvents ${ }^{\circledR}$ for its implementation.

Note that the above list of library components is non-exhaustive and might need to be extended depending on the peripherals that are available on the used microcontroller and the used communication protocol.

\subsection{Using the Library to Model an Embedded Platform}

The components described in Sections 3.1 and 3.2 are stored in a library and provided as a toolbox to the embedded engineer. It enables them to model an embedded platform, in which each microcontroller runs a particular RTOS, at a higher abstraction level. Note that due to the chosen abstraction level certain microcontroller aspects such as caches and pipelines are not explicitly modeled. They are contained in the configuration parameters that are related to the timing information. The model of the embedded platform allows engineers to execute a co-simulation with the modeled control algorithm and investigate to what extent the embedded platform affects the performance of the control algorithm. In order to obtain this co-simulation framework, the following design steps should be followed:

\section{Encapsulate the control algorithm's subsystems as Simulink ${ }^{\circledR}$ functions}

Each subsystem of the control algorithm that will be executed by a software task or ISR must be transformed to a global Simulink ${ }^{\circledR}$ function.

\section{Model the embedded platform}

Using the library blocks. The RTOS blocks of section 3.1 are used to model the appropriate RTOS, whilst the hardware blocks of section 3.2 are used to model the microcontroller's peripheral devices.

\section{Configure the embedded platform}

The execution times, resource usage, event usage and other properties of the software tasks and ISRs are configured using their block parameters. Then, in the Task Processor block, for each segment of the tasks, one calls the corresponding Simulink ${ }^{\circledR}$ function created in the first step.

Once the framework is modeled and configured, the plant model can be incorporated such that a MiL simulation can be executed. While observing the simulation results it is verified whether the specifications are satisfied. If not, the framework allows the engineers to easily evaluate different alternatives by reconfiguring 
task parameters (e.g., task priorities), review the model of the control algorithm, etc. If all specifications are met, the control algorithm can be deployed on the embedded platform.

\section{4 (Semi-)Automatic Deployment on the Embedded Platform}

As we already mentioned, our library consists of blocks that model software and hardware components of a specific embedded platform. In particular, the RTOS library blocks are models of the OSEK/VDX specification. This allows us to partially automate the deployment process as some information of the embedded platform, such as the number of tasks and their priorities, is already configured in the model. Trampoline (Bechennec et al. 2006), a RTOS that implements the OSEK/VDX specification, relies on OSEK Implementation Language (OIL) files to describe the entire configuration of the RTOS. Using a model-to-text transformation our framework enables engineers to extract the information contained in the co-simulation framework in order to automatically generate the required OIL file for Trampoline. At the time of writing, the code generation from the control model is still a separate activity that has to be initiated by the engineer using Simulink ${ }^{\circledR}$ Coder. However, the framework already generates templates that facilitates one to link the generated C-source files to the RTOS configuration.

\section{VALIDATION OF THE APPROACH}

We validate our approach using a case study of an electric power window. We compare regular MiL simulation results and the ones obtained when incorporating our models of the embedded platform with the results of the deployed system. For the latter, traces are obtained from a Hardware-in-the-Loop (HiL) simulation. Furthermore, we compare our framework with TrueTime, a state-of-the-art Simulink ${ }^{\circledR}$ toolbox that similarly introduces deployment effects in a MiL simulation.

\subsection{Setup}

Our electric power window case study consists of two windows located at the driver and passenger side of the vehicle. The driver is able to operate both windows, while the passenger is only capable of operating its own window. Driver operations have priority in case both operate the passenger's window. The power window case study is typed by a set of requirements, and derived specifications, as described in (Mosterman et al. 2004). In the scope of this validation, we are only interested in the two of them pertaining to an object being pinched between a closing window:

1. When closing the window, a force of no more than $120 \mathrm{~N}$ may be present.

2. If an object is detected while closing the window, it should seize moving up and lower by approximately $10 \mathrm{~cm}$.

Concerning the second requirement, we want to note how the spatial aspect of this requirement is not the most importance element. Instead, engineers will focus on the temporal aspect of this requirement, i.e., the time delta between applying the pinch, detecting the pinch and then stopping and lowering the window. After all, a life-threatening situation might occur if the window does not respond timely to a pinch.

For our comparison, we rely on models of an electric power window that are introduced in (Mosterman et al. 2004). This ensures that the controller operates correctly, and also ensures that the accompanying plant model is verified to be correct. The control algorithm is divided into subcomponents that are mapped onto a set of RTOS tasks distributed over two Atmel Atmega2560 microcontrollers, one for the driver side 
and one for the passenger side. The microcontrollers communicate with each other using a CAN bus, with a baud rate of $500 \mathrm{kbps}$, on which no other traffic is present. The task configuration of RTOS is listed below:

- Button Inputs - Executes as ISR. Microcontrollers: both. Reads the input signal (up/down).

- Debounce - Period: 10 ms. Microcontrollers: both. Debounces the input signals.

- Control Exclusion - Executes aperiodically. Microcontrollers: passenger side. Ensures the driver's inputs have priority over the passenger's.

- Power Window Control - Period: 25 ms. Microcontrollers: both. Controls one power window.

- CAN Tx - Period: 10 ms. Microcontrollers: driver side. Transmits driver inputs over the CAN bus.

- CAN Rx - Executes as ISR. Microcontrollers: passenger side. Receives the driver side inputs.

- Non-preemptable Task - Executes once at 2.9 seconds. Microcontrollers: both. This is a nonpreemptable task with a low priority. Non-preemptable tasks can be used for tasks that may have a low priority when scheduled but must run to completion without preemption once started. In this case that task simulates an operation that takes $400 \mathrm{~ms}$ to complete.

Note that the scheduling behavior of the components was validated in small test cases. As such, the last listed task is added to clearly show the scheduling effects in the simulations.

In order to evaluate our co-simulation framework, three simulations are executed:

1. A simulation of the regular control model and plant: this simulation sets a baseline for the expected control behavior. No tools or libraries are used, and as a result the impact of the non-preemptable task will not be visible. We refer to this simulation as MiL.

2. A HiL simulation for which the control model is deployed on the embedded platform while the plant models (one for each window) are executed on a real-time simulator. We refer to this simulation as HiL.

3. MiL simulations where tools/libraries are used to incorporate scheduling effects. We refer to these simulations as SimEvents ${ }^{\circledR}$ MiL, for the simulation using the library presented in this paper and TrueTime MiL for the simulation with TrueTime. The SimEvents ${ }^{\circledR}$ MiL simulation was built according to the steps described in section 3.3. The TrueTime MiL was built by converting the controller subsystems to Matlab ${ }^{\circledR}$ scripts. Note that TrueTime has no notion of non-preemptable tasks. However, we were able to achieve similar behavior by raising the priority of the non-preemptable task once it starts execution. T-Res posed the same problem, but unlike with TrueTime we were unable to implement a workaround. Additionally, the use of 4 triggered tasks for the button inputs was also not supported. As such we do not include a T-Res MiL simulation in the comparison.

\subsection{Results}

Figure 6 visualizes the position of the window. At the 3 second mark an object is pinched between the closing window and the door frame. Table 1 lists the measurements that have been taken to verify if the requirements are met. The regular MiL simulation passes both requirements: detecting the pinch and acting in a timely manner. The HiL simulation fails for requirement 1 due to the non-preemptable task hogging the processor. The SimEvents ${ }^{\circledR}$ MiL approximates the HiL well and also fails the first requirement. The TrueTime MiL initially shows similar behavior, however it deviates at the 4.1 second mark and fails both requirements.

When comparing the results of Table 1, we notice that simulation results using our library approximates the deployed behavior more accurately compared to the TrueTime simulation results. This can be explained by 


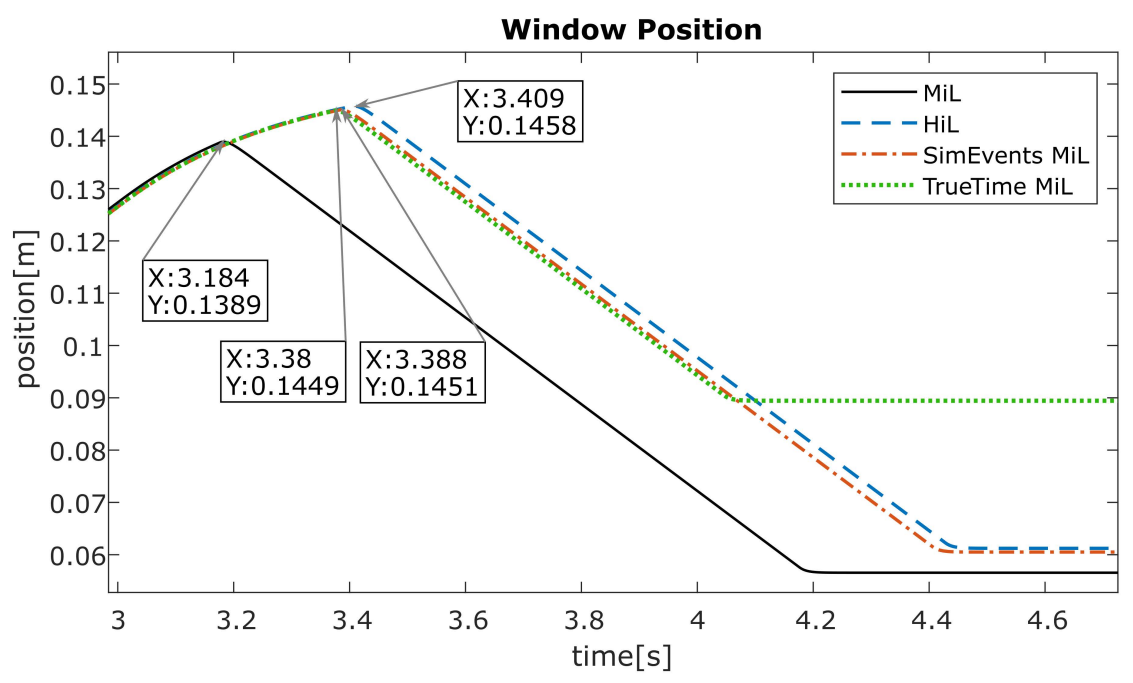

Figure 6: Driver side window position when detecting a pinched object.

Table 1: Simulation results for each simulation run.

\begin{tabular}{c|l|c|c|c|c} 
Req. & Measurement & MiL & HiL & SimEvents ${ }^{\circledR}$ MiL & TrueTime MiL \\
\hline $\mathbf{2}$ & Pinch detected after [ms]: & 150 & 383 & 372 & 371 \\
\hline $\mathbf{2}$ & Window seizes moving after [ms]: & 33 & 26 & 16 & 6 \\
\hline $\mathbf{1}$ & Maximum pinch force [N]: & 113.9 & 190.2 & 185 & 182.2 \\
\hline $\mathbf{2}$ & Window lowers by [cm]: & 8.25 & 8.45 & 8.45 & 5.5
\end{tabular}

the fact that our approach models a specific embedded platform. For example, the OSEK/VDX specific nonpreemptable task is not inherently supported by TrueTime. Through a workaround in the scripts, we were able to obtain a non-preemptable task. However, the TrueTime simulation still exhibits different behavior as it diverges at the 4.1 second mark, as can be seen in Figure 6. This is due to the fact that TrueTime doesn't allow the modeler to set the maximum number of activations of a task. So while the non-preemptable task is running, the other tasks are queued and activated repeatedly. As a result, once the non-preemptable task finished its execution, the queued tasks execute in rapid succession. Using the components of our library, one is able to set the maximum activation limit of the power window control task, which is set to 1 . As this corresponds to task settings of the deployed algorithm, the SimEvents ${ }^{\circledR}$ MiL simulation approximates the realized system more accurately. Thus, we conclude that the different behavior of the TrueTime simulation can be attributed to the fact that the TrueTime scheduler does not behave the same as the OSEK/VDX scheduler. Also, we observed that the configuration of a MiL simulation using the presented approach is significantly simpler compared to configuring an MiL simulation using TrueTime. Using the presented approach, the control and embedded engineer can follow the three steps described in section 3.3, after which the model is ready for simulation. When using TrueTime, the Stateflow ${ }^{\circledR}$ charts in the control model must be converted to scripts and the original control model must be significantly reworked in order to simulate the scheduling of the controller. In the presented approach the original control model must not be significantly reworked; i.e., subsystems must only be transformed to Simulink ${ }^{\circledR}$ functions.

\section{CONCLUSION AND FUTURE WORK}

We conclude by stating that using the presented library, the effects of deploying a control algorithm on hardware can be incorporated in a MiL simulation. We compared our approach with TrueTime, a state- 
of-the-art tool that similarly enables one to incorporate deployment effects in a control algorithm modeled with Simulink ${ }^{\circledR}$. When comparing our approach with TrueTime, we observed that the behavior of our cosimulation resembles the behavior of the deployed system more accurately while reducing the efforts for modeling the embedded platform. The more accurate results are obtained by providing library blocks that model a particular embedded platform. For example, instead of providing an RTOS block that abstracts a generic scheduler, we provide a scheduler that implements the OSEK/VDX specification. The reduced modeling efforts are obtained by minimizing the required efforts to transform a regular model of a control algorithm to one that can be co-simulated with the model of the embedded platform. While TrueTime focuses on a script-based configuration, our approach only requires one to transform ordinary subsystems to Simulink ${ }^{\circledR}$ functions. Additionally, the presented approach to incorporate deployment effects in a MiL simulation benefits both control and embedded engineer. The control engineer is able to evaluate and modify the behavior of his modeled algorithm as if it was deployed on the embedded platform, whilst the embedded engineer can $(i)$ alter the configuration of the embedded platform so that the control performance can be improved, and (ii) (semi-)automatically configure the embedded platform of the realized system as most information has already been provided in the model.

To complete this paper, we cover a couple of possibilities on future research directions. Firstly, the current RTOS library blocks are models (i.e., abstractions) of the OSEK/VDX specification. As such the simulation of other RTOS requires one to (partially) remodel the RTOS blocks. Expanding the library with more RTOS blocks opens up an interesting perspective, as it would allow the control engineer to switch out different RTOS blocks on the fly, in order to review their impact on the control algorithm. In that respect, it would be interesting to model a more complex RTOS such as RTLinux (Yodaiken and Barabanov 1997). Secondly, to achieve an accurate simulation, tasks and ISR need to have measured execution times. In this paper, we assumed these execution times could be obtained by deploying the control algorithm (without RTOS) and perform some time measurements. Instead, it may be more appropriate to deploy the algorithm on a cycle accurate simulator to obtain the execution times. This avoids the need for hardware to obtain the execution times. Thirdly, the current implementation allows to automatically generate the OSEK OIL configuration file from the co-simulation framework, while the $\mathrm{C}$-source code is generated in a template form. However, the Simulink ${ }^{\circledR}$ model does contain all the necessary information needed to automatically generate this source code. With that information in mind, an auto-generated binary executable from the control model opens up possibilities for future development.

\section{ACKNOWLEDGMENTS}

This work has been carried out within the CSE_CoDesigns_ICON project of the Flanders Make vzw.

\section{REFERENCES}

AUTOSAR 2018. “AUTOSAR - Enabling Innovation”. https://www.autosar.org/. Accessed Jul. 15, 2018.

Bechennec, J.-L., M. Briday, S. Faucou, and Y. Trinquet. 2006, sep. "Trampoline An Open Source Implementation of the OSEK/VDX RTOS Specification". In 2006 IEEE Conference on Emerging Technologies and Factory Automation, pp. 62-69, IEEE.

Brandberg, C., and M. Di Natale. 2018, jun. "A SimEvents Model for the Analysis of Scheduling and Memory Access Delays in Multicores". In 2018 IEEE 13th International Symposium on Industrial Embedded Systems (SIES), pp. 1-10, IEEE.

Cervin, A., D. Henriksson, B. Lincoln, J. Eker, and K.-E. Arzen. 2003. "How does control timing affect performance? Analysis and simulation of timing using Jitterbug and TrueTime". IEEE Control Systems vol. 23 (3), pp. 16-30. 
Cremona, F., M. Morelli, and M. Di Natale. 2015. "TRES: A Modular Representation of Schedulers, Tasks, and Messages to Control Simulations in Simulink". In Proceedings of the 30th Annual ACM Symposium on Applied Computing, SAC '15, pp. 1940-1947, ACM.

Denil, J., P. D. Meulenaere, S. Demeyer, and H. Vangheluwe. 2017. "DEVS for AUTOSAR-based system deployment modeling and simulation". SIMULATION vol. 93 (6), pp. 489-513.

Derler, P., E. A. Lee, S. Tripakis, and M. Törngren. 2013. "Cyber-physical system design contracts". In Proceedings of the ACM/IEEE 4th International Conference on Cyber-Physical Systems, pp. 109, ACM Press.

Henriksson, D., A. Cervin, and K.-E. Arzén. 2003. "TrueTime : Real-time Control System Simulation with MATLAB / Simulink". In Proceedings of the Nordic MATLAB Conference.

Lee, E. A. 2008. "Cyber physical systems: Design challenges". In Proceedings of the 11th IEEE Symposium on Object/Component/Service-Oriented Real-Time Distributed Computing.

Li, W., R. Mani, P. Mosterman, and T. Hubscher-Younger. 2016. "Simulating a Multicore Scheduler of RealTime Control Systems in Simulink". In 2016 Summer Computer Simulation Conference (SCSC 2016), Society for Modeling and Simulation International (SCS).

MathWorks 2019a. "Effects of Communication Delays on an ABS Control System". https://nl.mathworks. $\mathrm{com} / \mathrm{help} /$ simevents/examples/effects-of-communication-delays-on-an-abs-control-system.html.

MathWorks 2019b. "SimEvents”. https://nl.mathworks.com/products/simevents.html.

MathWorks 2019c. "Simulink". https://www.mathworks.com/products/simulink.

Mosterman, P., J. Sztipanovits, and S. Engell. 2004. "Computer-Automated Multiparadigm Modeling in Control Systems Technology”. IEEE Transactions on Control Systems Technology vol. 12 (2), pp. 223234.

OSEK/VDX 2005. "OSEK/VDX Operating System Specification 2.2.3 OSEK/VDX Operating System OSEK/VDX Operating System Specification 2.2.3”.

Vanherpen, K., J. Denil, H. Vangheluwe, and P. De Meulenaere. 2015. "Model Transformations for Roundtrip Engineering in Control Deployment Co-Design”. In TMS/DEVS '15, pp. 820-827, Society for Modeling and Simulation International (SCS).

Yodaiken, V., and M. Barabanov. 1997. “A Real-Time Linux”. Linux Journal vol. 34.

Zeigler, B. P. 1976. "Theory of Modeling and Simulation".

\section{AUTHOR BIOGRAPHIES}

JOOST MERTENS is a future doctoral student at the Faculty of Applied Engineering at the University of Antwerp. His email address is joost.mertens@student.uantwerpen.be.

KEN VANHERPEN is a post-doctoral researcher at the Faculty of Applied Engineering at the University of Antwerp. His email address is ken.vanherpen@uantwerpen.be.

JOACHIM DENIL is a professor at the Faculty of Applied Engineering at the University of Antwerp. His email address is joachim.denil@uantwerpen.be.

PAUL DE MEULENAERE is a professor at the Faculty of Applied Engineering at the University of Antwerp. His email address is paul.demeulenaere@uantwerpen.be. 